\title{
Simulação de um modelo de um sistema de resfriamento usando o software Aspen Plus ${ }^{\circledR}$
}

\author{
S. F. S. Vidal ${ }^{1}$, F. V. Silva ${ }^{2}$, I. C. Franco ${ }^{3}$ and A. M. F. Fileti ${ }^{4}$ \\ ${ }^{1,2,4}$ Universidade Estadual de Campinas, Faculdade de Engenharia Química, Departamento \\ de Sistemas Químicos e Informática \\ ${ }^{3}$ Centro Universitário da FEI
}

E-mail para contato: Saulo_vidal@hotmail.com

\begin{abstract}
RESUMO - Utilizando um modelo matemático derivado do software comercial Aspen ${ }^{\circledR}$, o comportamento dos estados estacionário e transiente de um sistema de refrigeração foi simulado com o objetivo de desenvolver uma ferramenta de análise do processo de refrigeração quando este fosse submetido a perturbações. Esse modelo foi parametrizado a partir da biblioteca de pacotes termodinâmicos e operações unitárias presentes no software. O programa computacional "Exchanger Design and Rating $®$ ", incluso no pacote Aspen $\AA$, foi empregado na modelagem da geometria dos trocadores de calor presentes no sistema de refrigeração. $\mathrm{O}$ modelo proposto representou efetivamente o protótipo experimental de refrigeração. A predição da performance do ciclo, assim como a análise dos impactos causados por perturbações, foi realizada eficientemente em torno da condição de operação. A simulação foi validada e está apta para explorar novos pontos de operação para esse sistema.
\end{abstract}

\section{INTRODUÇÃO}

A maioria dos processos químicas requer uma planta de refrigeração para que se possa resfriar ou manter em uma determinada temperatura uma corrente de processo, um produto, um equipamento, etc. A principal desvantagem desse tipo de sistema é alto gasto energético necessário. $\mathrm{O}$ desenvolvimento de modelos matemáticos e computacionais adequados para sistemas de refrigeração permite encontrar pontos de operação onde a eficiência do processo seja melhorada, permitindo a avaliação da implementação de técnicas de controles avançadas. De acordo com Schurt et al. (2001), condicionadores de ar e refrigeradores industriais consomem cerca de $10 \%$ da energia elétrica produzida em todo mundo. O alto consumo de energia envolvido nos processos de refrigeração tem motivado a produção de inúmeros estudos visando à análise de comportamento desses sistemas com o intuito de garantir uma maior eficiência durante a operação. Na literatura é possível encontrar diversos trabalhos de simulação de sistemas de refrigeração a partir de modelos computacionais, como Cabello et al. (2005), que desenvolveram um modelo bem simplificado, baseado em dados físicos e empíricos, capaz de predizer as condições de operação de um sistema de refrigeração a partir das temperaturas 
de condensação, evaporação e superaquecimento, mas que não leva em conta a dinâmica do processo.

No trabalho de Schurt et al. (2001), foi realizada uma modelagem matemática de um protótipo experimental de um sistema de refrigeração com o intuito de se avaliar o desempenho de um controle PID multivariável, fazendo uso de uma linearização do modelo em torno do ponto operacional, o que justifica o modelo se mostrar eficiente apenas para condições de operação próximas do ponto de operação. Uma revisão sobre técnicas de simulação computacional para problemas envolvendo sistemas de refrigeração por compressão é apresentada por Ding (2007).

O objetivo desse trabalho é parametrizar um modelo computacional para que este seja capaz de simular um sistema de refrigeração e analisar sua dinâmica com o intuito de discutir seu comportamento e possíveis formas de controle. As operações unitárias e as correlações termodinâmicas foram obtidas a partir da biblioteca do Aspen ${ }^{\circledR}$. Esse software é capaz de simular processos químicos com alta confiabilidade, seja em regime permanente ou transiente. Dados experimentais de um protótipo de refrigeração foram usados para construir e validar o modelo.

\section{PROTÓTIPO EXPERIMENTAL}

O protótipo experimental de uma planta de refrigeração localizado no Laboratório de Controle e Automação de Processos (LCAP) da Faculdade de Engenharia Química da Unicamp pode ser visto na Figura 1.

\subsection{Ciclo De Fluido Primário}

O ciclo primário conta com o fluido refrigerante R22 (fluido primário) circulando pelos componentes e removendo calor do fluido a ser refrigerado (fluido secundário) e fornecendo calor através do condensador.

O compressor usado nesse sistema é o modelo 2FC2.2 Bitzer Compressores ${ }^{\circledR}$ com dois cilindros, aberto, de alta velocidade e para altas, médias e baixas temperaturas. A velocidade de rotação do mesmo pode ser ajustada através de um conversor de frequência.

A válvula de expansão termostática é a mais comumente utilizada em plantas de refrigeração, tendo a função de regular a vazão de líquido refrigerante no evaporador em proporção ao superaquecimento do vapor na linha de sucção. É usual instalar válvulas de expansão termostática em sistemas de refrigeração para garantir o mínimo superaquecimento estável no evaporador.

Tanto para as unidades do evaporação e condensação foi utilizado um trocador de calor do tipo placas, modelo CB 26M Alfa Laval ${ }^{\circledR}$. O design desse modelo oferece altas taxas de transferência de calor pela circulação de fluidos alternadamente entre suas placas. 


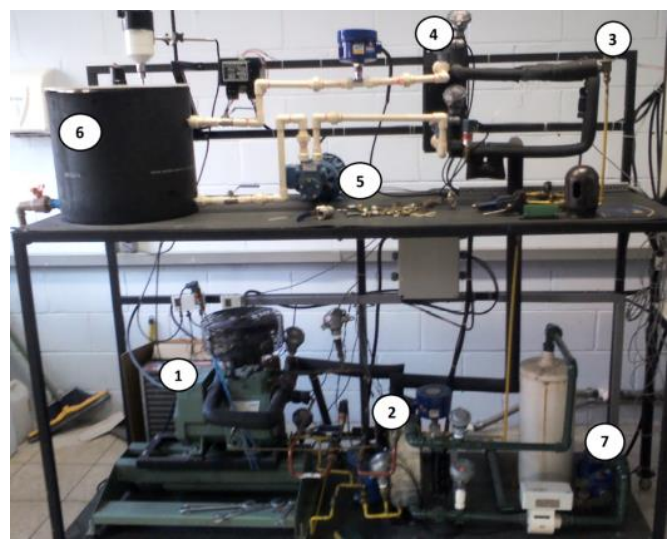

Figura 1 - Protótipo experimental do sistema de refrigeração. 1-Compressor; 2-Condensador; 3-Válvula de expansão; 4-Evaporador; 5-Bomba da linha fluido secundário; 6-Tanque de armazenamento da solução de propileno glicol; 7-Bomba da linha de condensação

\subsection{Ciclo De Fluido Secundário}

Na linha de fluido secundário foi utilizada uma solução aquosa de propileno glicol 50\% (vol.) como o fluido a ser refrigerado pelo sistema. Foi utilizada uma solução de propileno glicol pelo fato dessa congelar a temperaturas em torno de $-50^{\circ} \mathrm{C}$.

Para promover a circulação do fluido secundário através do ciclo foi utilizada uma bomba de deslocamento positivo, modelo RZR500, com um motor de 0,75 HP. A rotação dessa bomba foi manipulada com um conversor de frequência. Um resistor elétrico feito de aço tubular (reforçado, $220 \mathrm{~V}, 4000 \mathrm{~W}$ ) foi acoplado a um tanque de $60 \mathrm{~L}$ de estocagem de propileno glicol com o objetivo de simular a carga térmica do sistema. Um agitador a velocidade constante garante a homogeneização da temperatura do fluido dentro do tanque.

\subsection{Torre De Resfriamento}

Para garantir uma operação estável no condensador e uma maior economia no consumo de água, foi construída uma torre de resfriamento. A torre opera com contracorrentes de ar e água. A bomba usada para impelir a água através do condensador é uma bomba centrífuga KSB Hydrobloc P500, de 500 HP, 220 V e com vazão máxima de 2400L/h

\section{MODELAGEM COMPUTACIONAL}

A simulação estacionária e dinâmica dos ciclos descritos foram realizadas usando os softawares Aspen Plus ${ }^{\circledR}$ e Aspen Dynamics ${ }^{\circledR}$, respectivamente. O software Exchanger Design and Rating (EDR)® foi previamente usado para se obter a geometria do trocador de placas, a fim de seu comportamento dinâmico possa ser levado em conta na simulação dinâmica no Aspen Dynamics®. O último programa foi utilizado para implementar as perturbações e avaliar o comportamento do sistema global.

\subsection{Modelagem Dos Trocadores De Calor}


As condições de entrada e saída das correntes quente e fria, assim como as vazões mássicas e pressões foram fornecidas ao EDR para que se pudesse determinar a geometria dos trocadores de calor. Uma vez que os dois trocadores de calor são idênticos entre si, apenas um dos trocadores foi modelado pelo programa, e a mesma geometria foi utilizada no Aspen Plus ${ }^{\circledR}$ para modelar o evaporador e o condensador. Os dados alimentados no programa e mostrados na Tabela 1 foram obtidos de um experimento no protótipo citado e são relativos à unidade de condensação. Após determinada a geometria do trocador, o modelo foi salvo com extensão EDR e exportado para o Aspen Plus®.

Tabela 1- Dados de operação do condensador utilizados nos modelos dos trocadores de calor

\begin{tabular}{|c|c|c|c|c|}
\hline \multirow{3}{*}{ Variáveis } & \multicolumn{2}{|c|}{ Fluido Quente } & \multicolumn{2}{|c|}{ Fluido Frio } \\
\hline & Entrada & Saída & Entrada & Saída \\
\hline & \multicolumn{2}{|c|}{ R22 } & \multicolumn{2}{|c|}{ Solução $50 \%$ de propileno glicol } \\
\hline Vazão mássica $\left(\mathrm{kg} / \mathrm{m}^{3}\right)$ & \multicolumn{2}{|c|}{120,0} & \multicolumn{2}{|c|}{800,0} \\
\hline Temperatura $\left({ }^{\circ} \mathrm{C}\right)$ & 70,0 & 39,2 & 31,3 & 32,3 \\
\hline Pressão (bar) & 13,8 & 12,4 & 6,0 & 5,4 \\
\hline
\end{tabular}

\subsection{Parametrização Do Sistema De Refrigeração}

$\mathrm{Na}$ parametrização do modelo do sistema de refrigeração foram adotadas algumas simplificações, que não comprometeram o desempenho do modelo:

a) Com exceção da linha de sucção, todas as perdas de pressão na tubulação foram desconsideradas. A queda de pressão e a mudança de temperatura podem ser significantes e estão relacionadas ao superaquecimento do evaporados na linha de sucção.

b) Foi considerado que os acessórios do ciclo, como o tanque de separação de óleo e os sensores, não influenciavam significativamente no modelo.

Para cada equipamento foi selecionado um bloco específico no software Aspen Plus®. As variáveis de entrada para cada bloco foram determinadas a partir de dados experimentais e de forma que não houvessem divergência entre elas. Os blocos foram interconectados através de correntes de material, disponíveis no Aspen Plus ${ }^{\circledR}$. Para caracterizar as condições de operação das correntes foram necessárias informações de temperatura, pressão, vazão e composição. As correntes caracterizadas dessa forma foram as correntes da linha de sucção (ciclo primário), da entrada do tanque (ciclo secundário) e da sucção na bomba da condensação linha de condensação. O fluxograma desenvolvido no Aspen Plus ${ }^{\circledR}$ pode ser visto na Figura 2. A Tabela 2 resume todos os dados inseridos no modelo. 


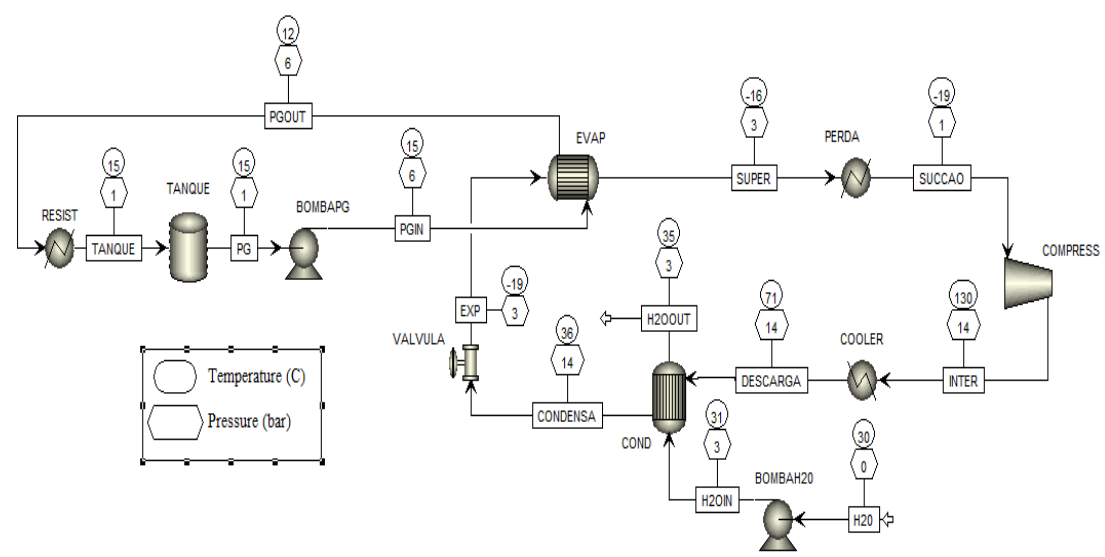

Figura 2 - Fluxograma desenvolvido no Aspen Plus® para modelar o protótipo de refrigeração

Tabela 2 - Dados de entrada no modelo

\begin{tabular}{|l|l|}
\hline Bloco/Corrente & \\
\hline SUCCAO & Temperatura: $1,4{ }^{\circ} \mathrm{C} ;$ Pressão: 1,43 bar; Vazão de R22: $73 \mathrm{~kg} / \mathrm{h}$ \\
\hline PGOUT & Temperatura: $6,8^{\circ} \mathrm{C}$; Fração de vapor: 0; Vazão: PG: $640 \mathrm{~kg} / \mathrm{h}, \mathrm{H} 2 \mathrm{O}: 1400 \mathrm{~kg} / \mathrm{h}$ \\
\hline COMPRESS & Tipo: isentrópico; curvas de performance \\
\hline COOLER & Pressão (negativo significa perda de carga): $-0,0001$ bar; Taxa de calor: $-1086,58 \mathrm{~W}$ \\
\hline COND & Modo de cálculo: Pratos; graus de subresfriamento da corrente quente: $3^{\circ} \mathrm{C}$ \\
\hline VALVULA & Pressão de saída: 2,9 bar \\
\hline EVAP & Modo de cálculo: Pratos; graus de superaquecimento da corrente fria: $6{ }^{\circ} \mathrm{C}$ \\
\hline PERDA & Pressão: 1,4 bar; Taxa de calor: 136,99 W \\
\hline BOMBAH2O & Potência: $0,5 \mathrm{Hp}$ \\
\hline BOMBAPG & Potência: $0,75 \mathrm{Hp}$ \\
\hline TANQUE & Pressão: 1,0 bar; Fases válidas: apenas líquida \\
\hline RESIST & Pressão: 1,1 bar; Taxa de calor: 3 kW; Fases válidas: líquida apenas \\
\hline
\end{tabular}

\section{RESULTADOS E DISCUSSÃO}

\subsection{Validação}

Para validar o modelo proposto, foram realizados experimentos sob condições diversas com o intuito de comprovar a acurácia do modelo em diferentes pontos de operação. Em cada experimento, o sistema foi perturbado e alcançou outro estado estacionário. As perturbações foram 
aplicadas na carga térmica ou na frequência de rotação do compressor. As variáveis observadas foram a temperatura de entrada e saída do evaporador da corrente contendo a solução de propileno glicol e a temperatura de evaporação do refrigerante. Gráficos de dispersão compararam os dados experimentais com os valores encontrados pelo modelo, como mostrado na Figuras 4. Foi realizada uma regressão linear para avaliar a acurácia do modelo. Como nos três casos o coeficiente angular foi muito próximo de 1 , o erro do modelo pode ser considerado como aproximadamente o valor do coeficiente linear, que teve seu máximo valor em $0,80^{\circ} \mathrm{C}$. Como o erro médio experimental (erro dos termopares) é de $0,50^{\circ} \mathrm{C}$, o erro do modelo é muito bem aceitável.

a)

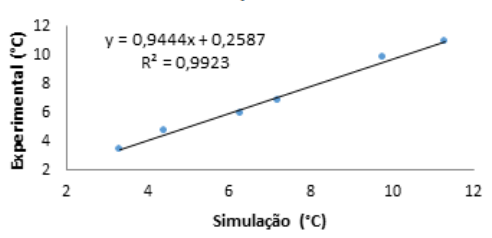

b)

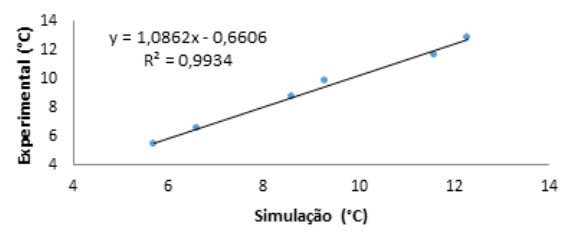

c)

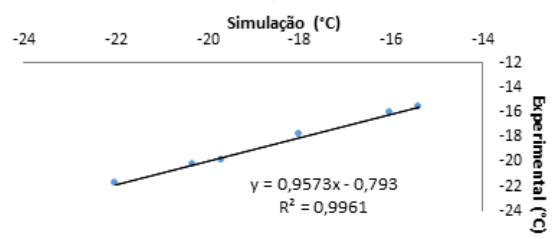

Figura 4 - Comparação entre dados experimentais e simulados: a) temperatura de saída da solução de propileno glicol do evaporador; b) temperatura de entrada da solução de propileno glicol do evaporador e c) Temperatura de evaporação do fluido refrigerante: comparação entre dados experimentais e simulados.

\subsection{Comportamento Dinâmico Do Sistema De Refrigeração}

Para se avaliar a linearidade do sistema, degraus positivos e negativos foram impostas nas variáveis independentes: rotação da bomba de fluido secundário, rotação do compressor e carga térmica. As duas primeiras foram escolhidas por serem possíveis variáveis manipuladas do processo, enquanto que a última é um distúrbio comum em sistemas de refrigeração. Os degraus foram aplicados sempre em estado estacionário. As condições iniciais de estado estacionário foram de $55 \mathrm{~Hz}$ de rotação do compressor e bomba, e $2 \mathrm{~kW}$ para a carga térmica. A Figura 9 (a) mostra o comportamento da temperatura da solução de propileno glicol à saída do evaporador sob perturbações da ordem de $\pm 10 \%, \pm 15 \%$ e $\pm 20 \%$ na rotação do compressor. Os valores dos ganhos são plotados na Figura 9 (b), e é possível perceber a não-linearidade entre as variáveis para pontos mais afastados do ponto de operação.

A Figura 10 (a) mostra os valores de ganho do processo quando a variável perturbada é a carga térmica e a variável observada continua sendo a temperatura da solução de propileno glicol à saída do evaporador. As perturbações foram da ordem de $\pm 10 \%, \pm 25 \%$ e $\pm 35 \%$. Neste caso, um comportamento próximo do linear pode ser observado e pode-se dizer que o ganho é de aproximadamente $2,45^{\circ} \mathrm{C} / \mathrm{kW}$. A Figura 10 (b) apresenta os ganhos entre a mesma variável observada para perturbações na rotação da bomba. As perturbações foram da ordem de $\pm 10 \%$, $\pm 15 \%$ e $\pm 20 \%$. Um comportamento próximo do linear também foi observado neste caso, apresentando um ganho em torno de $0,16^{\circ} \mathrm{C} / \mathrm{Hz}$. 


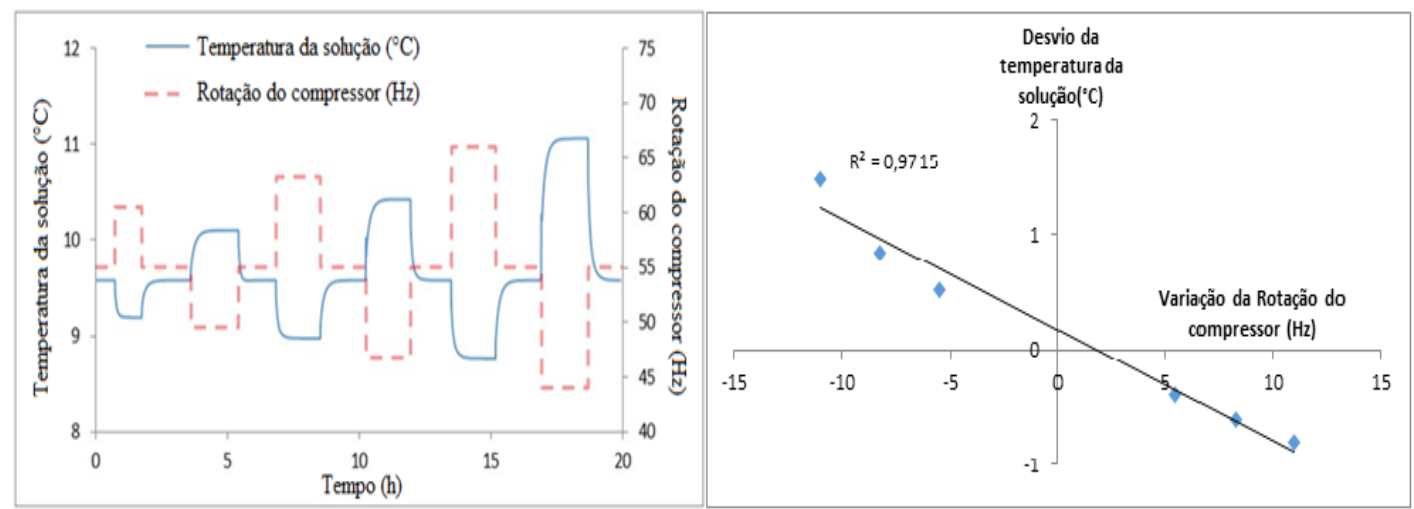

Figura 9 - a) Comportamento da temperatura do propileno glicol à saída do evaporador frente a perturbações na rotação do compressor; e b) valores de ganho entre as variáveis manipulada e observada

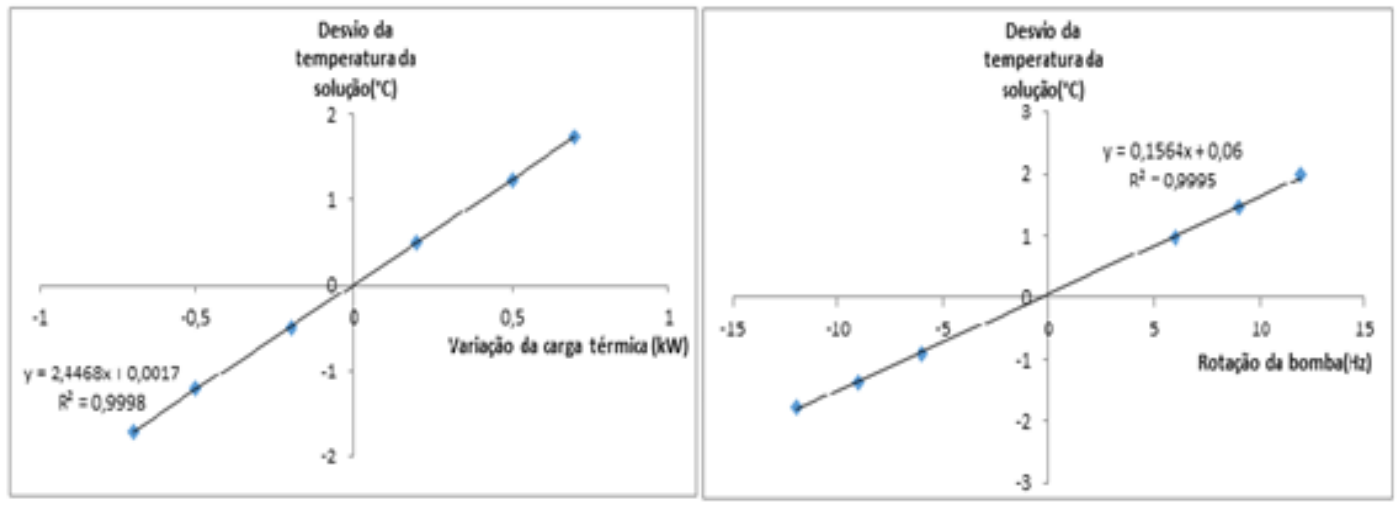

Figura 10 -a) valores de ganho entre a carga térmica e a variável observada; e b) valores de ganho entre a rotação da bomba e a variável observada

A Figura 11 mostra o diagrama de blocos em malha aberta do processo em termos de variável desvio, sendo Wb'(s) a frequência de rotação da bomba (em Hz), Q'(s) a carga térmica do sistema (em kW), e Wc'(s) a frequência de rotação do compressor. Os valores das constantes de tempo estão dadas em minutos.

A importância de se conduzir estudos acerca da linearidade do sistema está relacionada com a escolha da estratégia de controle apropriada. Conhecendo-se o a dinâmica do sistema é possível predizer o comportamento da variável controlada, tornando mais fácil o desenvolvimento de uma malha de controle preditivo. Se o sistema for predominantemente não linear, uma estratégia de controle inteligente deve ser empregada preferencialmente. É importante ressaltar que todas as conclusões foram verificadas para o protótipo experimental em condições em torno do ponto de operação. Contudo, a ferramenta desenvolvida está pronta para investigar condições adversas do sistema proposto, avaliando a performance do sistema e eficiência energética do mesmo. 


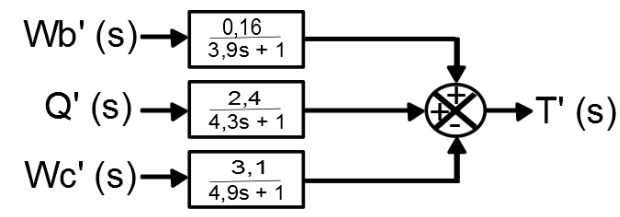

Figura 11 - Diagrama de blocos do processo em malha aberta

\section{CONCLUSÃO}

O modelo parametrizado a partir do pacote Aspen ${ }^{\circledR}$ se mostrou eficiente na representação do sistema de refrigeração experimental proposto, se tornando, portanto, uma importante ferramenta na determinação do comportamento dinâmico do sistema.

A relação não linear entre a temperatura à saída do evaporador da solução de propileno glicol e a rotação do compressor indica que, para fins de controle desse processo, uma estratégia de controle clássica pode não ser adequada quando as condições de processo desviam significantemente da condição inicial. Por outro lado, a rotação da bomba de fluido secundário pode ser manipulada eficientemente para o controle da mesma variável citada, uma vez que a dependência entre elas é linear. $\mathrm{O}$ conhecimento de como as perturbações podem afetar a variável monitorada permite a modelagem de estratégias de controle preditivo. Para o sistema de refrigeração proposto, considerando a carga térmica como o distúrbio, um modelo linear preditivo seria adequado para controle da temperatura.

Dada a complexidade de sistemas de refrigeração e a dificuldade de se realizar medidas das variáveis na planta experimental (relacionadas principalmente ao desgaste dos equipamentos e ruídos), o modelo desenvolvido irá desempenhar um importante papel na escolha de estratégias de controle, inclusive multivariável. Índices de performance, como COP e gasto energético, podem ser facilmente computados através da simulação, permitindo a avaliação de desempenho energético de cada proposta de controle e sintonia.

\section{REFERENCIAS}

A.C. CLELAND. RADS - a computer package for refrigeration analysis, design and simulation. Int. J. Refrigeration, v. 8, p. 372-373, 1985.

G. DING. Recent developments in simulation techniques for vapour-compression refrigeration systems. Int.l J. Refrigeration, v. 30, p. 1119-1133, 2007.

L.C. SCHUR; C.J.L. HERMES; A.T. NETO. Assessment of the controlling envelope of a modelbased multivariable controller for vapor compression refrigeration systems. Appl. Thermal Engineering, v. 30, p. 1538-1546, 2001.

L. HUA; S.K. JEONG; S.S. YOU. Feedforward control of capacity and superheat for a variable speed refrigeration system. Appl. Thermal Engineering, v. 29, p. 1067-1074, 2009.

R. CABELLO; J. NAVARRO; E. TORRELA. Simplified steady-state modelling of a single stage vapour compression plant. Model development and validation. Appl. Thermal Engineering, v. 25, p. 1740-1752, 2005. 